\title{
AN OPERATIONAL MODEL FOR THE OPTIMAL OPERATION OF THE FREEZE-DRYING PROCESS
}

\author{
Carlos Vilas $^{1, *}$, Antonio A. Alonso ${ }^{1},{\text { Eva Balsa } \text { Canto }^{1}, \text { Estefanía López Quiroga }^{2} \text {, Cristian Trelea }}^{3}$ \\ ${ }^{1}$ Grupo de Ingeniería de Procesos (IIM-CSIC), Eduardo Cabello, 6, Vigo (Spain), carlosvf@iim.csic.es \\ ${ }^{2}$ School of Chemical Engineering, University of Birmingham, Edgbaston, Birmingham, B152TT (UK) \\ ${ }^{3}$ INRA, Genie \& Microbiol Proc. Alimentaires UMR782, F-78850 Thiverval Grignon, (France)
}

\begin{abstract}
In this work an operational mathematical model for the freeze-drying process is derived. The model describes the state variables related to product quality and stability. Computational issues associated to the presence of a moving front are approached by using the Landau transform. Unknown model parameters are estimated using experimental data. The model is used to design optimal operation policies that reduce the process duration ensuring product quality.
\end{abstract}

Keywords: Lyophilization; Landau transform; Parameter estimation; Dynamic optimization; Moving boundary.

\section{INTRODUCTION}

Freeze-drying, or lyophilization, is a dehydration process used to preserve quality attributes of the material to be dried. In this process water is first frozen and then sublimated by lowering temperature and pressure. Low temperatures prevent any chemical or biological deterioration of the product. This technology has been considered in food industry to maintain the organoleptic and nutritional properties of the foodstuff. Transport is also easier because, since water is removed, the resulting product is much lighter. Unfortunately, freeze-drying is a costly process both in terms of the energy and process time. Hence, its use in food or biotechnology industry is restricted to the production of high value products [10, 3. One typical application, which motivated the present research, is the supply of high quality bacteria cultures employed as starters in cheese manufacture 9 .

Nonetheless, as it has been discussed in 7 for instance, there exists plenty of room for improvements in freeze-drying operation. The authors demonstrated, on a simulation basis, significant reductions in process costs and improvements in quality when using optimal operation profiles.

Recently, most research efforts have been concentrated on the development of control schemes to compute and to implement optimal time-varying operating profiles 14. Such profiles can be updated on-line in response to unexpected process disturbances, or to variability in the properties of the material or initial conditions. To that purpose, reliable descriptions of the process in the form mathematical models are required.

There is a long modeling tradition for freezedrying (e.g. 6, 8]). Classical models typically incorporate all heat and mass transfer mechanisms covering the whole range of space and time scales, from the shortest (mass transfer in the dried media) usually operating in the order of microseconds, to the slowest with time constants in the order of hours (e.g diffusive heat transfer in both the frozen and dried phases). In general such models are complex due to the varied range of time and spatial scales considered which results into large simulation times. This precludes its use in real time operation where quick simulations are required in order to produce, in due time, optimal decisions. In addition they usually include a large number of parameters difficult to be estimated from the available experimental information.

In this work we present a simple model able to capture the observed dynamics of the system. One critical aspect of the model that hampers its numerical solution lies in the coexistence of two phases (dried and a frozen) and a front evolving in the material. We overcome this by using the so-called Landau transform which sets a fixed location of the front [5, 4. Parameter estimation is performed from input-output data records obtained with standard sensing devices, available in most commercial equipments. Besides, the process is split into two decoupled subsystems, namely product and condenser, to simplify the model identification problem. Finally, the model is used to compute optimal operation policies that significantly reduce process time while ensuring product stability and quality. 


\section{FREEZE-DRYING PROCESS PHYSICAL MODEL}

Freeze-drying is a dehydration process which consists of the following three stages: (i) Freezing. During this stage the free water in the product is frozen. Ice crystals growth is controlled to avoid possible damage to the food or biological material. (ii) Primary drying. This stage conditions most of the quality properties of the product. Ice is sublimated by gradually heating the product under very low pressure conditions (always below the triple point). The water leaves the product in the form of vapor. Product temperature is usually kept below a critical value, which depends on the product, to avoid collapse. (iii) Secondary drying. In this stage bound water is removed from the product by desorption.

Primary drying is a complex stage that involves heat and mass transfer mechanisms, as well as ice sublimation. The following, widely employed, assumptions are made to reduce model complexity: (i) the frozen region has uniform heat and mass transfer properties; (ii) a continuous interface with infinitesimal thickness is considered between the frozen and dried layers; (iii) the matrix pore structure is permeable to the vapor flux and it is not deformable.

A careful dimensionless analysis of the governing equations was performed in [7] and revealed several time-scales involved in the primary drying. This analysis allows us to focus just on the phenomenon of interest from the parameter estimation and optimal control points of view. The mechanisms occurring at time scales very different from the phenomenon of interest are neglected in order to simplify the model equations.

Parameters involved in the model are summarized in Table 1.

\subsection{THE PRIMARY DRYING}

Vapor flux contribution to the energy balance can be neglected as it is a much faster phenomenon than heat conduction. Focus will, therefore, be on heat conduction. In its $1 \mathrm{D}$ version, energy balance leads to (for details see [7]):

$$
\begin{aligned}
\rho_{d r} c_{p, d r} \frac{\partial T_{d r}}{\partial t} & =\kappa_{d r} \frac{\partial^{2} T_{d r}}{\partial \xi^{2}} \\
\rho_{f r} c_{p, f r} \frac{\partial T_{f r}}{\partial t} & =\kappa_{f r} \frac{\partial^{2} T_{f r}}{\partial \xi^{2}}
\end{aligned}
$$

where subindices $d r$ and $f r$ refer to dried and frozen region, respectively. $T(t, \xi)$ is the food product temperature and $\xi$ are the spatial coordinates (product height).
Note that, during primary drying, sublimation makes the front to move from the top to the bottom of the product resulting into a moving boundary problem. If we denote by $x(t)$ the front position, the spatial domains of the dried and frozen regions are defined as $\xi \in[0, x(t))$ and $\xi \in(x(t), L]$, respectively. The movement of the front is described using the Stefan condition [8]:

$$
w=\frac{\left(\left.\kappa_{f r} \frac{\partial T_{f r}}{\partial \xi}\right|_{\xi=x^{+}}-\left.\kappa_{d r} \frac{\partial T_{d r}}{\partial \xi}\right|_{\xi=x^{-}}\right)}{\left(\rho_{f r}-\rho_{d r}\right) \Delta H_{s}}
$$

where $w=\mathrm{d} x / \mathrm{d} t$ represents the front velocity.

Boundary conditions are required to solve Eqs (1)(3). At the product top $(\xi=0)$ heat is transported mainly by radiation. At the product bottom, radiation and conduction are modeled, via gas-surface collisions [10]. Therefore we have:

$$
\begin{gathered}
\left.\kappa_{d r} \frac{\partial T_{d r}}{\partial \xi}\right|_{\xi=0}=\sigma e_{p} f_{p}\left(T_{c h}^{4}-\left.T_{d r}\right|_{\xi=0} ^{4}\right) \\
\left.\kappa_{f r} \frac{\partial T_{f r}}{\partial \xi}\right|_{\xi=L}=h_{L}\left(T_{s h}-\left.T_{f r}\right|_{\xi=L}\right)
\end{gathered}
$$

where $T_{c h}$ and $T_{s h}$ are, respectively, the chamber and the shelf temperatures. Heat transfer coefficient $h_{L}$ takes the following form [10]:

$$
h_{L}=h_{L, 1}+\frac{h_{L, 2} P_{c h}}{1+\frac{P_{c h}}{h_{L, 3}}}
$$

with $P_{c h}$ being the total chamber pressure. $h_{L, 1}$ combines the contribution of conduction and radiation (after linearization). $h_{L, 2}$ and $h_{L, 3}$, representing the contribution of gas-surface collisions to the heat transfer, are given constant parameters.

At the front, continuity of temperature across the front is considered, i.e. $\left.T_{d r}\right|_{\xi=x(t)}=\left.T_{f r}\right|_{\xi=x(t)}=$ $T_{\text {front }}$. At this boundary, vapor and ice are assumed to be in equilibrium so that the front temperature $\left(T_{\text {front }}\right)$ can be computed from the front pressure using the Clausius-Clapeyron equation:

$$
T_{\text {front }}=\frac{1}{\frac{1}{273.11}-K_{\text {clap }} \log \left(\frac{P_{\text {front }}}{611.72}\right)}
$$

Front pressure $\left(P_{\text {front }}\right)$ is computed using Darcy's law [2] which, after a finite differences discretization may be expressed as:

$$
P_{\text {front }}=P_{c h}+K_{c} w\left(\rho_{f r}-\rho_{d r}\right)
$$

where

$$
K_{c}=k_{1} P_{c h}+k_{2} x
$$

with $k_{1}$ and $k_{2}$ being given parameters. 
Table 1: Parameters involved in the freeze-drying model. Parameters to be estimated are indicated in the Value column as t.b.e.

\begin{tabular}{|c|c|c|c|}
\hline Parameter & Value & Units & Description \\
\hline$\rho_{d r}$ & 200.31 & $\mathrm{~kg} \mathrm{~m}^{-3}$ & Dried region density \\
\hline$\rho_{f r}$ & 1001.6 & $\mathrm{~kg} \mathrm{~m}^{-3}$ & Frozen region density \\
\hline$c_{p, d r}$ & 1254 & $\mathrm{~J} \mathrm{~kg}^{-1} \mathrm{~K}^{-1}$ & Dried region heat capacity \\
\hline$c_{p, f r}$ & 1818.8 & $\mathrm{~J} \mathrm{~kg}^{-1} \mathrm{~K}^{-1}$ & Frozen region heat capacity \\
\hline$\kappa_{d r}$ & t.b.e. & $\mathrm{Wm} \mathrm{m}^{-1} \mathrm{~K}^{-1}$ & Dried region heat conductivity \\
\hline$\kappa_{f r}$ & 2.4 & $\mathrm{~W} \mathrm{~m}^{-1} \mathrm{~K}^{-1}$ & Frozen region heat conductivity \\
\hline$L$ & $5.75 \times 10^{-3}$ & $\mathrm{~m}$ & Food product height \\
\hline$\sigma$ & $5.6704 \times 10^{-8}$ & $\mathrm{~W} \mathrm{~m}^{-2} \mathrm{~K}^{-4}$ & Stefan-Boltzmann constant \\
\hline$e_{p}$ & 0.78 & - & Thermal emissivity at the product top \\
\hline$f_{p}$ & 0.99 & - & Geometrical correction factor \\
\hline$K_{\text {clap }}$ & $1.6548 \times 10^{-4}$ & $\mathrm{~K}^{-1}$ & Constant in the Clapeyron equation \\
\hline$\Delta H_{s}$ & $2791.2 \times 10^{-3}$ & $\mathrm{~J} \mathrm{~kg}^{-1}$ & Sublimation heat \\
\hline$R$ & 8314 & $\mathrm{~Pa} \mathrm{~m}^{3} \mathrm{~K}^{-1} \mathrm{kmol}^{-1}$ & Ideal gas constant \\
\hline$D_{z}$ & 0.242 & $\mathrm{~m}$ & Food product length \\
\hline$D_{y}$ & 0.307 & $\mathrm{~m}$ & Food product width \\
\hline$M_{w}$ & 18 & $\mathrm{~kg} \mathrm{kmol}^{-1}$ & Water molecular mass \\
\hline$h_{L, 1}$ & t.b.e. & $\mathrm{W} \mathrm{m}^{-2} \mathrm{~K}^{-1}$ & Heat transfer coefficient constant \\
\hline$h_{L, 2}$ & t.b.e. & $\mathrm{W} \mathrm{m}^{-2} \mathrm{~K}^{-1} \mathrm{~Pa}^{-1}$ & Heat transfer coefficient constant \\
\hline$h_{L, 3}$ & 34.4 & $\mathrm{~Pa}$ & Heat transfer coeff. constant \\
\hline$k_{1}$ & t.b.e. & $\mathrm{s} \mathrm{m} \mathrm{kg}^{-1}$ & Mass transfer coeff. constant in Darcy's equation \\
\hline$k_{2}$ & t.b.e. & $\mathrm{sPakg}^{-1}$ & Mass transfer coeff. constant in Darcy's equation \\
\hline$\beta$ & t.b.e. & $\mathrm{kg} \mathrm{s}^{-1}$ & Mass transfer coeff. constant in chamber/condenser flux \\
\hline$T_{c h}$ & 293.15 & $\mathrm{~K}$ & Chamber temperature \\
\hline$V_{c h}$ & 0.202 & $\mathrm{~m}^{3}$ & Chamber volume \\
\hline$\tau_{A}^{r e f}$ & $2.689 \times 10^{4}$ & $\mathrm{~S}$ & Compartment A reference time constant \\
\hline$\tau_{B}^{r e f}$ & $6.493 \times 10^{5}$ & $\mathrm{~S}$ & Compartment B reference time constant \\
\hline$E_{a}$ & $4.271 \times 10^{4}$ & $\mathrm{~kJ} \mathrm{~kg}^{-1}$ & Activation energy \\
\hline$\alpha_{A}$ & 0.669 & - & Compartment $\mathrm{A}$ ratio between equilibrium water contents \\
\hline$\alpha_{B}$ & 0.331 & - & Compartment B ratio between equilibrium water contents \\
\hline$M_{g}$ & 0.0434 & $\mathrm{~kg}$-water $\mathrm{kg}^{-1}$-total & Constant of the GAB equation \\
\hline$C_{g}$ & 7.4789 & - & Constant of the GAB equation \\
\hline$K_{g}$ & 0.9827 & - & Constant of the GAB equation \\
\hline$K_{T, g}$ & 8.2 & - & Constant of the glass transition temperature \\
\hline$T_{g, l}$ & 138.15 & $\mathrm{~K}$ & Constant of the glass transition temperature \\
\hline$T_{g, s}$ & 348.75 & K & Constant of the glass transition temperature \\
\hline
\end{tabular}

\subsection{THE SECONDARY DRYING}

At this stage of the process there is no frozen water. Bounded water is eliminated by desorption. The spatial domain is fixed during secondary drying so classical numerical methods can be employed to solve the model equations. Eq (1) with boundary conditions (4)- (5), where $\xi \in[0, L]$, is used to describe product temperature evolution and distribution during this stage.

\subsection{THE CONDENSER}

A condenser is used to remove, from the chamber, part of the vapor produced by ice sublimation. Accumulation of vapor in the chamber is computed as:

$$
\frac{\mathrm{d} m_{c h}^{v}}{\mathrm{~d} t}=\phi_{p}^{v}-\phi_{c}^{v}
$$

where $\phi_{p}^{v}$ denotes the vapor flux from the product to the chamber and coincides with the flux of ice sublimated:

$$
\phi_{p}^{v}=\left(\rho_{f r}-\rho_{d r}\right) D_{z} D_{y} \frac{\mathrm{d} x}{\mathrm{~d} t}
$$

Flux from the chamber to the condenser $\left(\phi_{c}^{v}\right)$ can be described using the classical theory of binary diffusion in gases which leads to [15]:

$$
\phi_{c}^{v}=\beta \ln \left(\frac{P_{c h}-P_{c}^{v}}{P_{c h}-P_{c h}^{v}}\right)
$$

Also, from the ideal gas equation, we have:

$$
\frac{\mathrm{d} P_{c h}^{v}}{\mathrm{~d} t}=\frac{R T_{c h}}{M_{w} V_{c h}} \frac{\mathrm{d} m_{c h}^{v}}{\mathrm{~d} t}
$$

where $M_{w}$ denotes the molecular weight of water.

\subsection{DESORPTION MODEL}

The desorption model is used to determine both final water content in the product (related to product quality) and the glass transition temperature 
(related to product stability). The content of bound water in the product $\left(c_{w}\right)$ is expressed as the ratio between mass of bound water $\left(m_{w}\right)$ and total mass (bound water plus solids):

$$
c_{w}=\frac{m_{w}}{m_{w}+m_{s}}
$$

Water is assumed to be distributed between two "compartments" (compartments A and B), corresponding to different physical states and/or interactions with the solid matrix (bacterial cell, membrane, cryoprotectant, etc.). Drying kinetics, per compartment, is given by [12]

$$
\frac{\mathrm{d} c_{w, i}}{\mathrm{~d} t}=-\frac{1}{\tau_{i}}\left(c_{w, i}-c_{w, i}^{e q}\right) ; \quad \text { with } i=A, B
$$

with $\tau_{i}$ being the desorption time function and $c_{w, i}^{e q}$ the equilibrium water content for compartment $i$. Desorption time constants are assumed to obey an Arrhenius-like relationship [13]:

$$
\tau_{i}=\tau_{i}^{r e f} \exp \left[\frac{E_{a}}{R}\left(\frac{1}{T_{p}}-\frac{1}{T^{r e f}}\right)\right]
$$

Equilibrium water content is computed as $c_{w, i}^{e q}=$ $\alpha_{i} c_{w}^{e q}$ with $\alpha_{A}+\alpha_{B}=1$. $c_{w}^{e q}$ is computed using the classical Guggenheim-Anderson-Boer (GAB) equation [9]:

$$
c_{w}^{e q}=\frac{M_{g} C_{g} K_{g} a_{w}}{\left(1+K_{g} a_{w}\right)\left(1+K_{g} a_{w}\left(C_{g}-1\right)\right)}
$$

where $a_{w}$ stands for water activity. Glass transition temperature is related to the water content as follows [1]:

$$
\begin{gathered}
T_{g}\left(c_{w}\right)=\frac{K_{T_{g}}\left(T_{g, 1}-273.15\right) c_{w}}{K_{T_{g}} c_{w}+\left(1-c_{w}\right)}+ \\
\frac{\left(1-c_{w}\right)\left(T_{g, s}-273.15\right)}{K_{T_{g}} c_{w}+\left(1-c_{w}\right)}
\end{gathered}
$$

As mentioned above, $T_{g}$ can used to predict product integrity. In this regard, if the glass transition temperature exceeds the product temperature in a given quantity the foodstuff will collapse.

\section{THE LANDAU TRANSFORM}

As mentioned above, the sublimation part of the process (primary drying) - see section 2.1 involves a moving boundary. In order to avoid the use of advanced numerical techniques, like the Arbitrary Lagrangian-Eulerian (ALE) algorithm [8], we apply the Landau transform [5]. As a result, we will obtain an equivalent system with fixed domain in which classical numerical techniques for the solution of partial differential equations can be applied. The moving boundary splits the spatial domain in two regions (dried and frozen) in which different transforms $\left(Q_{d r}, Q_{f r}\right)$ are defined.

$$
\begin{array}{r}
Q_{d r}: \xi \rightarrow z ; \quad\left\{z \in \mathbb{R} \mid z=\frac{\xi}{x}\right\} \\
Q_{f r}: \xi \rightarrow y ; \quad\left\{y \in \mathbb{R} \mid y=\frac{\xi-x}{L-x}\right\}
\end{array}
$$

Note that $z, y \in[0,1]$. Temperature in the new coordinates is denoted by $\mathcal{T}$, this is: $T_{d r}(t, \xi) \rightarrow$ $\mathcal{T}_{d r}(\theta, z), T_{f r}(t, \xi) \rightarrow \mathcal{T}_{f r}(\theta, y)$.

Eqs. (1) and (2) are expressed, in terms of $z, y$, as:

$$
\begin{gathered}
\frac{\partial \mathcal{T}_{d r}}{\partial \theta}=\frac{\alpha_{d r}}{x^{2}} \frac{\partial^{2} \mathcal{T}_{d r}}{\partial z^{2}}+\frac{z w}{x} \frac{\partial \mathcal{T}_{d r}}{\partial z} \\
\frac{\partial \mathcal{T}_{f r}}{\partial \theta}=\frac{\alpha_{f r}}{(L-x)^{2}} \frac{\partial^{2} \mathcal{T}_{f r}}{\partial y^{2}}+w \frac{1-y}{L-x} \frac{\partial \mathcal{T}_{d r}}{\partial y}
\end{gathered}
$$

where $\alpha_{i}=\kappa_{i} /\left(\rho_{i} c_{p, i}\right)$ with $i=d r, f r$.

Note that, in order to solve (17) and (18), two phases are required. In other words, front position must be $0<x(t)<1$ for all times. Boundary conditions -Eqs (4) and (5)- as well as Stefan equation (3) must be also expressed in terms of the new coordinate system with fixed domain $(z, y)$ :

$$
\begin{aligned}
& \left.\frac{\kappa_{d r}}{x} \frac{\partial \mathcal{T}_{d r}}{\partial z}\right|_{z=0}=\sigma e_{p} f_{p}\left(T_{c h}^{4}-\left.\mathcal{T}_{d r}\right|_{z=0} ^{4}\right) \\
& \left.\frac{\kappa_{f r}}{L-x} \frac{\partial \mathcal{T}_{f r}}{\partial y}\right|_{y=1}=h_{L}\left(T_{s h}-\left.\mathcal{T}_{f r}\right|_{y=1}\right) \\
& w=\frac{\left(\left.\frac{\kappa_{f r}}{L-x} \frac{\partial \mathcal{T}_{f r}}{\partial y}\right|_{y=0}-\left.\frac{\kappa_{d r}}{x} \frac{\partial \mathcal{T}_{d r}}{\partial z}\right|_{z=1}\right)}{\left(\rho_{f r}-\rho_{d r}\right) \Delta H_{s}}
\end{aligned}
$$

Figure 1 presents the results obtained with the Landau technique (marks) and the ALE method (lines). The latter was implemented in a specialized software (COMSOL, www.comsol.com). The

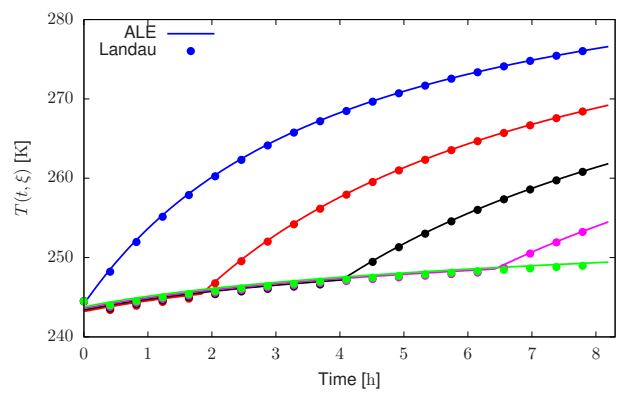

Figure 1: Freeze-drying model solution comparison.

comparison is performed in terms of the product temperature time evolution at five points equidistributed along the spatial domain. The figure illustrates that both techniques are equivalent. 
For comparison purposes we have employed parameters $\kappa_{d r}=0.0129, k_{1}=4.75 \times 10^{3}, k_{2}=$ $6.051 \times 10^{7}, h_{L, 1}=3.85, h_{L, 2}=0.352$, in addition to those of Table 1. Control variables are $T_{s h}=283.15 \mathrm{~K}, T_{c h}=293.15 \mathrm{~K}$ and $P_{c h}^{v}=20 \mathrm{~Pa}$.

\section{STRATEGIES FOR PARAMETER IDENTIFICATION}

Figure 2 depicts a schematic representation of the freeze-drying model. Freeze-drying process is split

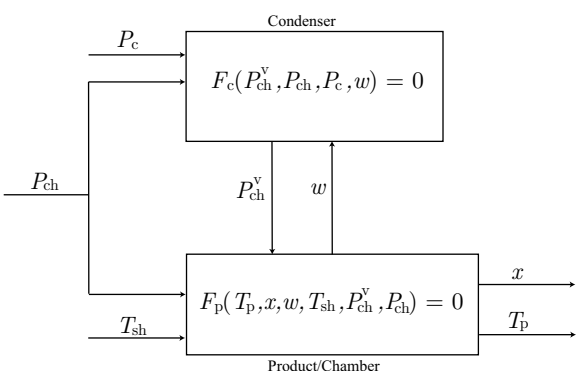

Figure 2: Schematic representation of the freeze drying process. Two subsystems are considered, the condenser and the product.

into two subsystems, the condenser and the product, each of them with an associated mathematical mode. Three controls are available in the pilot plant: condenser pressure $\left(P_{c}\right)$, chamber pressure $\left(P_{c h}\right)$ and shelf temperature $\left(T_{s h}\right)$. For estimation purposes condenser pressure is kept constant during the process. These variables are also measured.

Chamber vapor pressure $\left(P_{c h}^{v}\right)$ is the only state in the condenser model. Note that $P_{c h}^{v}$ is indirectly measured through the Pirani pressure $\left(P^{p}\right)$ [15]:

$$
P_{c h}^{v}=\frac{P^{p}-P_{c h}}{\alpha-1} ; \quad \alpha=1.6
$$

State variables in the food product are: front position $(x)$ and velocity $(w)$ as well as product temperature $\left(T_{p}\right) . T_{p}$ is only measured at a given spatial location which is not known with precision.

Condenser and product subsystems are coupled through $P_{c h}^{v}$ and $w . P_{c h}^{v}$ is measured, thus it can be used as an input to the product model decoupling. In other words, $P_{c h}^{v}$ measurements can be used to simulate the product part without using the condenser model. In this regard, the estimation task will proceed in the following two steps: (i) Estimation of the unknown parameters involved in the product model, using measurements of $P_{c h}^{v}$. (ii) Estimation of the unknown parameters involved in the condenser model.

\subsection{Parameter estimation for the product/chamber model}

Model parameters to be estimated are $\kappa_{d r}, k_{1}, k_{2}$, $h_{L, 1}$ and $h_{L, 2}$. Product temperature at a given spatial location $\left(T_{p}\left(\xi_{j}, t\right)\right)$ is measured. Sensor location might change among experiments. Cost function for parameter estimation is defined as:

$$
J=\frac{\sum_{k=1}^{n_{\text {exp }}} \sum_{i=1}^{n_{s, k}} \sqrt{\left(\bar{T}_{p, k}\left(t_{i}\right)-T_{p, k}\left(\xi_{j}, t_{i}\right)\right)^{2}}}{n_{s, k} n_{\exp }}
$$

subject to the model dynamics. In Eq. 22 $n_{\text {exp }}$ is the number of experiments and $n_{s, k}$ is the number of sampling times in experiment $k . \bar{T}_{p, k}\left(t_{i}\right)$ represents the product temperature measurement at time $t_{i}$ whereas $T_{p, k}\left(\xi_{j}, t_{i}\right)$ represents the model prediction of the product temperature at the spatial position $\xi_{j}$ and at time $t_{i}$.

\subsection{Parameter estimation for the condenser model}

Model parameter to be estimated here is $\beta$. In the plant, it is possible to experimentally measure the chamber total and Pirani pressures so that the chamber vapor pressure $\left(P_{c h}^{v}\right)$ can be obtained 15]. Cost function is, therefore, defined as:

$$
J=\frac{\sum_{k=1}^{n_{\exp }} \sum_{i=1}^{n_{s, k}} \sqrt{\left(\bar{P}_{c h, k}^{v}\left(t_{i}\right)-P_{c h, k}^{v}\left(t_{i}\right)\right)^{2}}}{n_{s, k} n_{\text {exp }}}
$$

subject to the model dynamics. $\bar{P}_{c h, k}^{v}\left(t_{i}\right)$ and $P_{c h, k}^{v}\left(t_{i}\right)$ are, respectively, the chamber vapor pressure measured and predicted by the model.

Parameter estimation tasks have been performed using the AMIGO toolbox [1].

\section{RESULTS AND DISCUSSION}

\subsection{PILOT PLANT AND EXPERIMENTAL SETUP DESCRIPTION}

Experiments were carried out on a LyoBeta special freeze-dryer equipped with two thermocouples, a capacitive manometer and a Pirani gauge. Lactic acid bacteria were produced by fermentation in controlled conditions of $\mathrm{pH}$ and temperature [9].

The following experimental protocol was applied for the shelf temperature: (i) Freezing at $223.15 \mathrm{~K}$ (cooling rate of $0.6 \mathrm{~K} \mathrm{~min}^{-1}$ ). (ii) Primary drying where temperature varies from $223.15 \mathrm{~K}$ to either $253.15,273.15$ or $293.15 \mathrm{~K}$ depending on the experiment. (iii) Secondary drying where temperature varies from primary drying temperature to 
Table 2: Optimal value for the parameters corresponding to the product subsystem.

\begin{tabular}{llllll}
\hline & $k_{1}$ & $k_{2}$ & $h_{L, 1}$ & $h_{L, 2}$ & $\kappa_{d r}$ \\
\hline Value & $4.27 \times 10^{2}$ & $7.83 \times 10^{7}$ & 3.34 & 1.62 & $8.92 \times 10^{-4}$ \\
Units & $\mathrm{m}^{-1}$ & $\mathrm{~Pa} \mathrm{~m}^{-2}$ & $\mathrm{~W} \mathrm{~m}^{-2} \mathrm{~K}^{-1}$ & $\mathrm{~W} \mathrm{~m}^{-2} \mathrm{~K}^{-1} \mathrm{~Pa}^{-1}$ & $\mathrm{~W} \mathrm{~m}^{-1} \mathrm{~K}^{-1}$ \\
\hline
\end{tabular}

298.15 K. Total chamber pressure was kept at 20 or $60 \mathrm{~Pa}$ depending on the experiment.

\subsection{MODEL IDENTIFICATION}

The identification protocol described in Section 4 will be applied to the pilot plant described above. Six experiments were carried out. Five of them were used for parameter estimation whereas the other was left for validation.

\subsubsection{The product-chamber subsystem}

Product temperature was measured by a thermocouple located at the bottom of the product. Time interval between two measurements is $1 \mathrm{~min}$. Unfortunately, due to experimental limitations, only an estimation of sensor position was available. For this reason, sensor location was included as an extra parameter to be estimated. Note that this parameter is experiment dependent.

Minimization of $J$ in Eq. 22 resulted into the parameter values summarized in Table 2. The values found are in accordance with the expected physical range. As mentioned above, sensor is intended to be located at the bottom of the food product, i.e. $L=5.75 \times 10^{-3} \mathrm{~m}$ (see Table 1 ). Estimation of sensor location resulted in $\xi_{s}=$ $5.18 \times 10^{-3} \mathrm{~m}$ for experiments $1,2,3$ and validation; $\xi_{s}=4.60 \times 10^{-3} \mathrm{~m}$ for experiment 4 and $\xi_{s}=5.75 \times 10^{-3} \mathrm{~m}$ for experiment 5 . All of them are close to the product bottom as expected.

Figure 3 represents experimental data (blue circles) vs. model predictions (blue line) for a fitting experiment and the validation experiment. The control temperature (black line) and the simulated product temperature at different spatial points (gray lines) are also depicted in the figure. Figure at the left corresponds to a fitting experiment whereas the validation experiment is represented in the figure at the right. Note that the end of the primary drying stage as well as the product temperature during both primary and secondary drying are predicted by the model with a reasonable degree of accuracy. However, some mismatch between predicted and measured temperatures have been observed in the transition from primary to secondary drying (highlighted in Figure 3 by two vertical black lines). So far no clear explanation has been found to such disagreement although it most probably can be attributed to: (i) movement
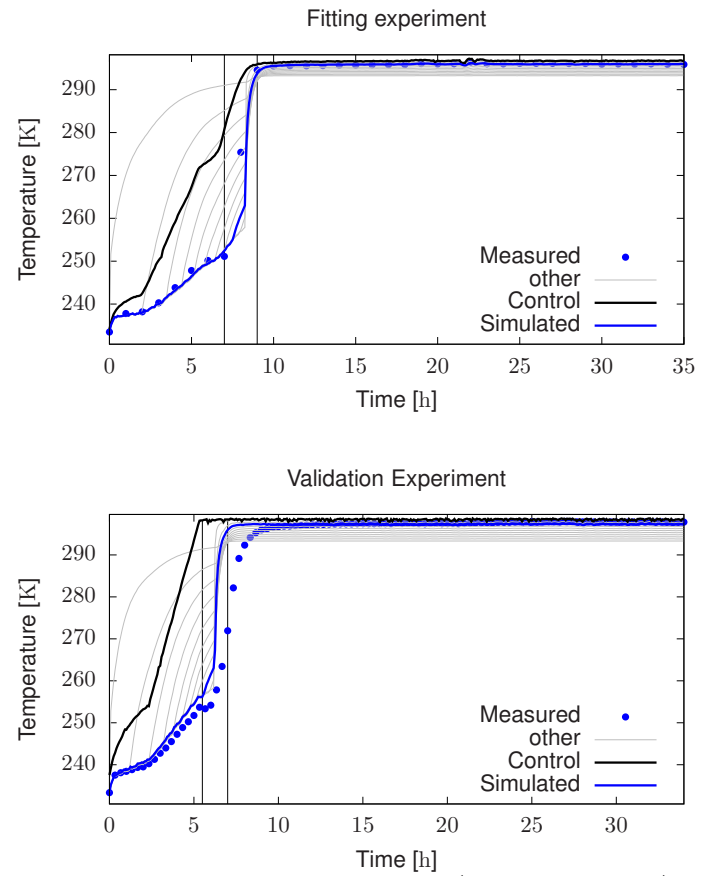

Figure 3: Freeze-drying model (food product) fitting and predictive capabilities. Vertical lines represent the end of primary drying and the beginning of secondary drying.

of the sensor after primary drying; or (ii) an interphase structure which is more complex than the sharp transition between a frozen and a dried region as it is considered by the model. Nevertheless, this mismatch does not have a significant impact on product quality.

\subsubsection{The chamber-condenser subsystem}

Measured variable is the chamber pressure. Minimization of $J$ in Eq. 23 led to $\beta=1.61 \times 10^{-6}$ $\mathrm{kg} \mathrm{s}^{-1}$. Predictive capabilities of this model are illustrated in Figure 4. Blue line and blue circles represent, respectively, model simulation results and experimental measurements for the chamber vapor pressure. Black line is the measured total pressure. Only primary drying is represented in the figures since during secondary drying vapor pressure is almost zero. As shown in the figure, the model offers a satisfactory degree of accuracy.

\subsection{DYNAMIC OPTIMIZATION OF THE FREEZE-DRYING PROCESS}

The objective of dynamic optimization is to find the shelf temperature time profile that minimizes 

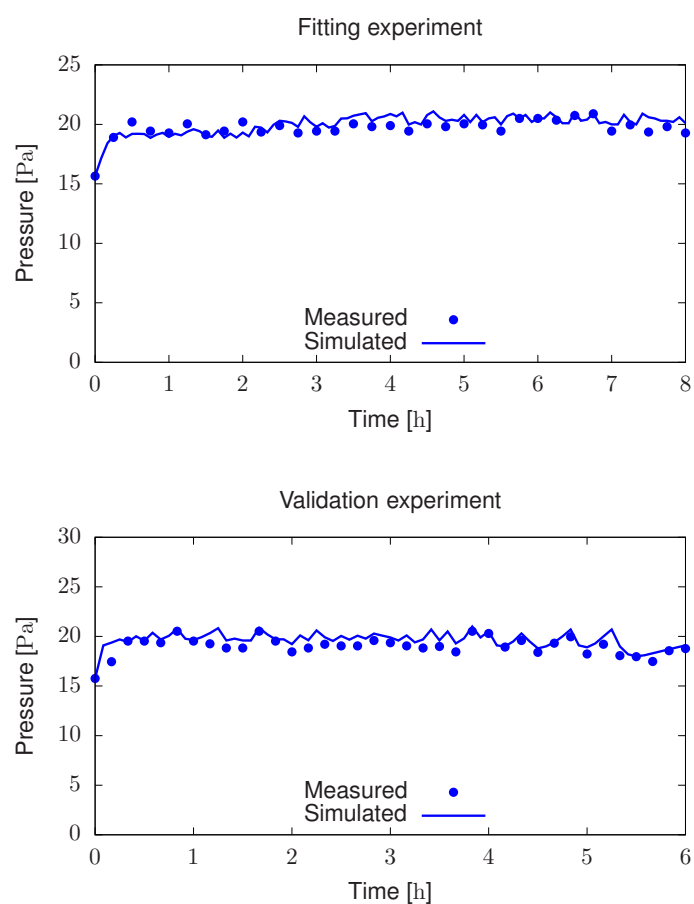

Figure 4: Freeze-drying model (condenser) fitting and predictive capabilities.

the process duration while fulfilling product quality and stability constraints. In this regard, note that large values for shelf temperature might result into product collapse. Glass transition temperature, see Eqn (14), is used to define two constraints to avoid collapse: the first one limits the maximum difference between product and glass transition temperatures. The second one prevents the system to be close to $T_{p, \max }$ for large periods.

$$
\begin{gathered}
T_{p}(\xi, t)-T_{g}(\xi, t) \leqslant T_{p, \max } \\
\int_{\tau}\left(T_{p}(\xi, t)-T_{g}(\xi, t)\right) \mathrm{d} t \leqslant I_{p, \max }
\end{gathered}
$$

Integration interval $(\tau)$ corresponds to the part of the time domain where $T_{p}>T_{g}$. The values $T_{p, \max }=307 \mathrm{~K}$ and $I_{p, \max }=124 \mathrm{Kh}$ were obtained by simulation of the first experiment.

Final product quality is related to final water content in the product. To ensure quality, the following constraint is considered:

$$
\int_{\xi} c_{w}\left(\xi, t_{f}\right) \mathrm{d} \xi \leqslant c_{w, \max }
$$

$c_{w, \max }=0.017 \frac{\mathrm{kg} \text { water }}{\mathrm{kg} \text { total }}$, taken from the final water content in the first experiment, is used.

Figure 5 represents the evolution of product temperature (gray lines on the top figures), product water content (bottom figures) and shelf temperature (black lines on the top figures). As expected, the shelf profile on the optimally designed experiment (see right column subplots) starts at a low value, to avoid product damage, and increases as the product becomes more stable. This profile is more aggressive than the one in experiment 1 . Final time is reduced by a $20 \%(8.3 \mathrm{~h})$. Product integrity and quality are ensured because constraints are fulfilled.

\section{CONCLUSIONS}

In this work we derived and validated an operational model describing the behavior of the freezedrying process. The model focuses on the time scale of the state variables related to product stability and quality, i.e. product temperature and water content.

Landau transform was used to obtain an equivalent system with fixed domains. This allowed us to avoid the use of specialized simulation software difficult to combine with decision making tools.

Model identification from data measured with standard sensing devices was carried out. To facilitate this task, the process was split in two subsystems (product and condenser). Then we solved a (decoupled) sequence of parameter identification problems, with a reduced number of unknown parameters. Model predictive capabilities have been tested on a validation experiment. Primary and secondary drying stages were accurately described by the model. However, some mismatch between predicted and measured temperatures were found at the transition between stages. This did not result into significant impact on product quality.

Finally, the model was employed to design optimal operation policies aiming at reducing process duration. Simulation tests showed that the optimal policy reduced process time in around $20 \%(8.3$ h) while keeping quality and stability standards.

\section{Acknowledgement}

The authors acknowledge the financial support received from E.U. H2020 research and innovation programme (CoPro project, No 723575) and E.U. $7^{\text {th }}$ Framework Programme (CAFE Project, KBBE-2007-2-3-01).

\section{References}

[1] E. Balsa-Canto and J. R. Banga. Amigo, a toolbox for advanced model identification in systems biology using global optimization. Bioinformatics, 27(16):2311-2313, 2011.

[2] G. Dagan. The generalization of Darcy's law for nonuniform flows. Water Resour. Res., 15(1):1-7, 1979. 

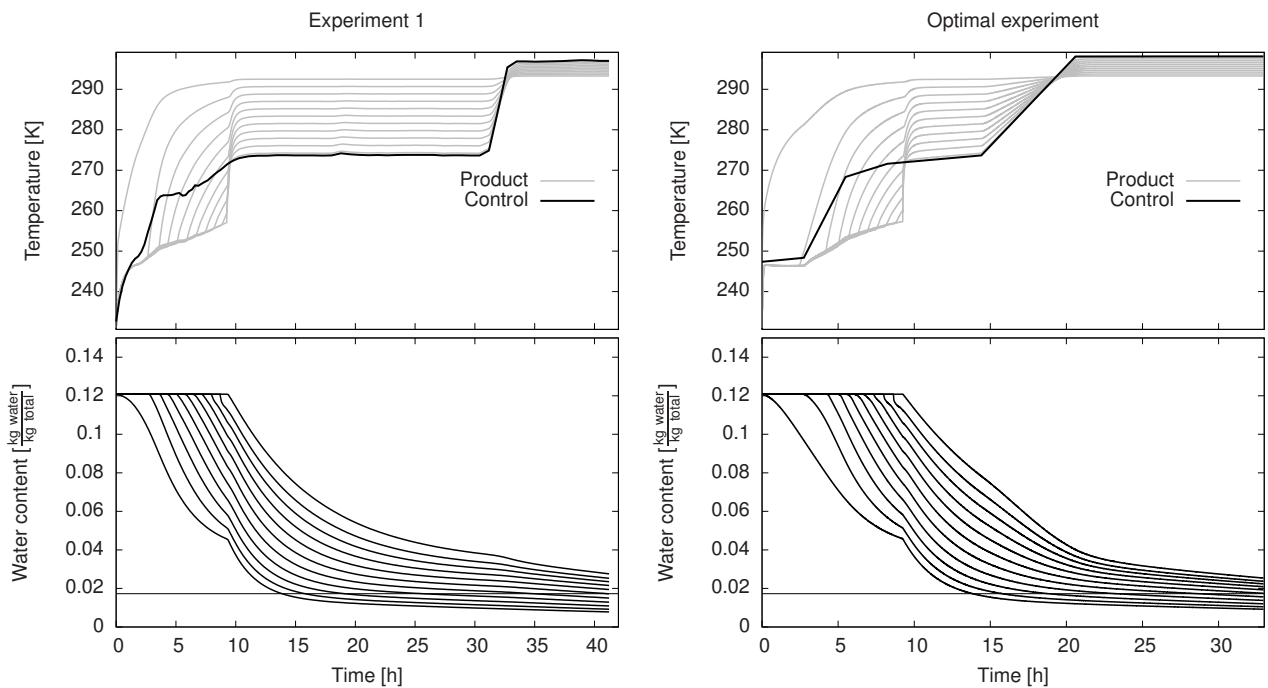

Figure 5: Comparison between a typical freeze-drying experiment (left figures) and the optimal one (right figures). Horizontal line on the bottom figures represent the constraint on the final water content.

[3] Z. Huifen, Y. Sheng, W. Dexi, L. Huixing, C. Xiaozhen, and Y. Lijun. Model of heat and mass transfer during vacuum freeze-drying for cornea. Math. Probl. Eng., ID941609:1$16,2012$.

[4] T. C. Illingworth and I. O. Golosnoy. Numerical solutions of diffusion-controlled moving boundary problems which conserve solute. $J$. Comput. Phys., 209(1):207-225, 2005.

[5] H. G. Landau. Heat conduction in a melting solid. Q. Appl. Math., 8(1):81-94, 1950.

[6] R. J. Litchfield and A. I. Liapis. An adsorption-sublimation model for a freezedryer. Chem. Eng. Sci., 34(9):1085-1090, 1979.

[7] E. Lopez-Quiroga, L. T. Antelo, and A. A. Alonso. Time-scale modeling and optimal control of freeze-drying. J. Food Eng., 111(4):655-666, 2012.

[8] W. J. Mascarenhas, H. U. Akay, and M. J. Pikal. A computational model for finite element analysis of the freeze-drying process. Comput. Meth. Appl. Mech. Eng., 148(12):105-124, 1997.

[9] S. Passot, S. Cenard, I. Douania, I. C. Trelea, and F. Fonseca. Critical water activity and amorphous state for optimal preservation of lyophilized lactic acid bacteria. Food Chem., 132(4):1699-1705, 2012.

[10] M. J. Pikal. Heat and mass transfer in low pressure gases: Applications to freeze drying. In: Amidon, G. L., Lee, P. I., Topp, E. M., editors. Transport processes in pharmaceutical systems. Drugs and the pharmaceutical sciences, Marcel Dekker, New York,2000.
[11] M. J. Pikal and S. Shah. The collapse temperature in freeze drying: dependence on measurement methodology and rate of water removal from the glassy phase. Int. J. Pharm., 62(2-3):165-186, 1990.

[12] M. J. Pikal, S. Shah, and M. L. Roy. The secondary drying stage of freeze drying: drying kinetics as a function of temperature and chamber pressure. Int. J. Pharm., 60(3):203$217,1990$.

[13] R. Pisano, D. Fissore, and A. A. Barresi. Quality by design in the secondary drying step of a freeze-drying process. Dry. Technol., 30(11-12):1307-1316, 2012.

[14] R. Pisano, D. Fissore, and A. A. Barresi. Inline and off-line optimization of freeze-drying cycles for pharmaceutical products. Dry. Technol., 31(8):905-919, 2013.

[15] I. C. Trelea, F. Fonseca, S. Passot, and D. Flick. A mutual gas diffusion model improves the prediction of mass transfer in freeze-drying. IDS 2014 - 19th Int. Drying Symposium, August 24-27, Lyon, France, 2014.

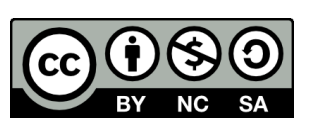
(c) 2019 by the authors. Submitted for possible open access publication under the terms and conditions of the Creative Commons Attribution CC BY-NC-SA 4.0 license (https://creativecommons.org/licenses/by-ncsa/4.0/deed.es). 